\title{
Blockchain-based online education content ranking
}

\author{
Anuj Garg ${ }^{1}$ - Sharmila A ${ }^{2}$. Pramod Kumar ${ }^{3}$ - Mani Madhukar ${ }^{4}$. \\ Octavio Loyola-González ${ }^{5} \cdot$ Manoj Kumar $^{6}$ (D)
}

Received: 1 April 2021 / Accepted: 2 November 2021 / Published online: 15 November 2021

(c) The Author(s), under exclusive licence to Springer Science+Business Media, LLC, part of Springer Nature 2021

\begin{abstract}
Nowadays, internet technology plays a vital role in all the fields of our daily lives ranging from the world economy, professional careers, higher education, and almost all the spheres that are deeply impacted. In the current situation, due to COVID19, the dependence on the Internet for almost everything, including learning, getting daily needs, etc., is heavily dependent on the Internet. Online learning is made possible by the Internet, and today most students, educators, researchers are leveraging online learning platforms to enhance their knowledge at their own pace. Generally, the quality of the E-learning courses is evaluated with the help of the courses' review and rating mechanisms. In the present context, review systems are centralized, storing highly valuable information at one location and are liable to manipulation, hacking, and tampering. In this paper, the Blockchain-based Online Education Content Ranking system is proposed for an online review and ranking system that offers a decentralized trustworthy system, ensuring the integrity of the rating and the independence and integrity of content reviews by Subject Matter Experts (SME).
\end{abstract}

Keywords E-learning · Blockchain Technology $\cdot$ SME · Instructional Design · Content Ranking

\section{Introduction}

Education is a key component for the sustainable growth of any civilization or country. The development and integration of various technologies have significantly impacted and contributed to making learning easy and meaningful for learners in the educational sector. The World Wide Web (WWW) outreach and the Internet have helped develop E-learning platforms to enhance the teaching and learning process effectively in the last decade. Even before COVID 19

Manoj Kumar

wss.manojkumar@gmail.com

Extended author information available on the last page of the article 
pandemic outbreak, global edtech investments reached US\$ 187.877 billion in 2019. COVID-19 pandemic outbreak has enforced a remarkable shift from traditional teaching to online teaching at schools and colleges worldwide. The online education global edtech investment is expected to reach US\$350 billion by 2025 . While the industry is snowballing and online education is getting more popular than traditional classroom education, there are many shortcomings in the current model (Al Harthy et al., 2019; Alammary et al., 2019; Chen et al., 2018a; Failed, 2019; Jansen et al., 2017; Kosba et al., 2016; Lizcano et al., 2019; Shaker et al., 2021; Sun et al., 2021; Williams, 2019; Yuan \& Wang, 2018).

In the present context, review systems are centralized, storing highly valuable information at one location and are liable to manipulation, hacking, and tampering. In this paper, the blockchain-based online education content ranking system is proposed which provides a decentralized online review system to validate the trustworthiness of the rating and enables the consortium-based onboarding of Subject Matter Experts (SME's) endorsed by the stakeholders to infuse independence and trust. Today's online rating system does not offer a Parity score. The course rating is not compared with other competitors in the E-learning platform to assist the user in understanding the benefits and shortcomings of similar courses available with different online course providers. In the proposed system, the parity score is recorded on the blockchain, ensuring the fudge-free rating. Also, blockchain-based meta-data ensures the integrity of the data items contributed to assessing the course's ranking.

Blockchain technology is being touted as the Internet of Trust that combats most of the issues of centralized data storage systems, including ranking manipulation and fudging of data. Blockchain implementation with a federated SME ranking system ensures the trustworthiness of a vendor's content, and it can trace the originality of the reviews and ratings. The Blockchain property of immutability and transparency ensures that the blockchain reviews are not being manipulated or deleted by any entity. The proposed online content rating system's unique feature is developed based on the Hyperledger fabric, a permissioned blockchain system. In brief, the proposed system's working can be explained as a blockchain-based novel rating system in which the rating information provided by independent SME's is stored and shared through the efficient and secure Hyperledger fabric network establishing parity. This system offers a user-friendly web interface for providing ratings and reviews of the course content. Further, the Hyperledger composer REST API is used to connect the web applications to the blockchain network. In this proposed system, the endorser performs the validation and authentication of the rating as defined in the smart contract's logic. The other advantage and unique feature of the proposed system is the federated rating. The federated rating is obtained by averaging the ratings provided by independent impaneled SMEs and stored in a blockchain-based ledger to infuse trust amongst owners of different online learning platforms. Accountability is a property of security notions to obtain guarantees. The proposed online content rating system is developed based on a permissioned blockchain system, where the misbehaving parties can be identified and held accountable. The Blockchain-based ledger will provide trust against any manipulations of rating, fudging of data, and 
bring transparency to the overall rating system. The main contribution of this paper is summarized as follows,

- The mechanism to onboard the content from various online content providers and rate the online education content from multiple online vendors through an independent SME consortium and creating Parity Score, using a decentralized Blockchain-based system.

- Define a rating system based on parameters agreed by content providers, rated by an independent SME panel, with a Federated score, leading to a new approach of ranking various courses in the same domain.

The remaining part of this paper is organized as follows in four further sections. Section II provides background information about blockchain and smart contracts. Section III details the related works. Section IV presents the proposed blockchainbased online education rating system. Section V summarizes the parameters used to analyze the performance of the proposed system. In the end, section VI provides the conclusion and future scope of the paper.

\section{Background}

This section elaborates on the essential background details such as blockchain, Hyperledger Fabric, and the role of Subject Matter Experts (SME) in the proposed online education content ranking.

Blockchain

Blockchain is regarded as the next-generation technology, also labeled as the Internet of Trust. It has presented the possibility of perceiving the Internet as a value rather than a medium of communication. While the first application of Bitcoin and the world of cryptocurrency continue to rule the world, the other applications of Blockchain technology will rule the next decade or so (Nakamoto and Bitcoin: A, 2008) (Nakamoto \& Bitcoin: A, 2008). The technology is growing at a rate of $80 \%$ CAGR and is expected to hold $10 \%$ of the world's GDP by 2025 (https://www.marke tsandmarkets.com, Market-Reports \& blockchain-technology-market-90, 100890. html. xxxx).

At its core, Blockchain is a distributed ledger that is not owned by a single party and is cryptographically secured using various crypto algorithms (Nakamoto \& Bitcoin: A, 2008). The Blocks store the transactions and information and they are chained to gather using cryptographic hashes. The technologies used in Blockchain contain some of the existing technologies like distributed ledger, asymmetric data encryption using PKI framework, consensus algorithms as Practical Byzantine Fault Tolerance, and message transfer protocols such as Whisper, etc. (Amin, 2020; Androulaki et al., 2018; Astarita et al., 2020; Azzi \& Chamoun, 2019; Chen et al., 2018b; Fernández-Caramés \& Fraga-Lamas, 2019; Hyperledger. Hyperledger Blockchain., 2020; Jiang et al., 2020; Sukhwani et al., 2018; Viriyasitavat \& Hoonsopon, 2019; Xu et al., 2018). 
While public Blockchains continue to rule the Blockchain market, the private or business Blockchains are emerging and seeing the practical usages now. Live applications can be seen in distributed supply chains, the healthcare industry, Government operations, and many other fields. The education sector is also coming up with many use cases, especially after the emergence of online players coming into the scene (Dentacoin, 2018; Revain, 2018; Beck et al., 2016; Cachin, 2016; Caliper, 2019; Hyperledger. Hyperledger Caliper., 2019; Lina.Review: Blockchain Based Review Platform \& LinaNetwork, 2018; Skiba, 2017; Swan, 2015; Zapit, 2018; Vukolić, 2015). Blockchain-based consortiums of educational institutes help for better collaboration and build up the grounds for more research-oriented higher education with collaboration from multiple institutes. Similarly, online content providers find synergies in collaborating via the Blockchain network and work effectively on common problems like effective content and federated ratings of the content.

The primary application of Blockchain technology in the business world consists of use cases where multiple business entities need to collaborate on specific, welldefined parameters and come to a standard agreement to devise the business outcomes (). Generally, in such situations, the intermediary is required to run the operations. Concerning the given context, multiple online education vendors can appoint an intermediary or an organization that can review the content, provide the rating for the content, and suggest improvements to make it more competitive and relevant for the market and industry. The key problem with having a middle man in such a situation is that they might incline towards giving favors and getting favorable ratings in return due to the various organizations' vested interests. This might deteriorate the situation instead of improving it from the current system of user ratings. The key question is that we can find a technology-inclined solution to get an honest rating that cannot be tampered with and can be stored in a secure database. The answer is Blockchain. In the present context, private / permissioned Blockchain is more suitable as the online education institutes' content is an intellectual property and cannot be shared on a public platform. To mitigate the risk of bias in the ranking system, the federated ranking system is suggested. It will decentralize the ranking process and avoid unbiased ranking.

Hyperledger fabric

Hyperledger (Caliper 2019) is an open-source blockchain platform developed by the Linux Foundation to endorse blockchain technology advancement. The Hyperledger platform identifies and addresses the significant features of a cross-sectoral open standard for the distributed ledger. Hyperledger fabric or simply fabric, is one of the projects of Hyperledger which IBM developed. Fabric architecture is designed to achieve a high degree of scalability, resilience, privacy flexibility, and confidentiality of the systems. The Hyperledger fabric platform is specifically suitable for an enterprise application. It allows implementing the chain code (smart contract) in any programming language (golang, Java, or Node.js) and running it in a Docker container. Fabric is a permissioned network that allows only valid/ registered users to participate in the transaction.

Fabric architecture is organized based on the service provided for the applications. Generally, it offers different services such as blockchain services, membership services, and chain code services. In the current proposal, since we have multiple 
participants with varied interests and participation principles, Hyperledger Fabric is the best-suited Blockchain implementation to support this use-case. Hyperledger fabric comes with a full permission system. It controls the level of access which is not possible in public blockchain. The proposed online rating system is based on Hyperledger fabric which provides distributed ledger and chain code functionalities as a service. The Hyperledger fabric ensures the security, integrity and prevents fake reviews and ranking of the online courses.

Role of subject matter expert (SME's)

Subject Matter Expert plays an important role in maintaining online courses' quality and ensuring the appropriate subject content used in the modules. The SME needs to be independent so that the ratings are not influenced also, the credentials of SME about his expertise need to be impeccable. SME inputs can help vendors enhance the subject content and guide the instructional designer of the course to design impactful and successful course content. The subject creator is a person who creates an e-content for the course. The content is reviewed by the independent SME and also ensures the appropriate ratings are given to the course content. Once the content is reviewed, the SME will suggest adding or removing the information from the course material and making interactive courses by providing the guidelines on when and where to include internal assessment throughout the course. SMEs review the content within a stipulated timeline and against specific parameters such as quality, duration, industry relevance, classification based on a beginner, Intermediator, expert, communication, ease of understanding, and practical orientation.

\section{Related works}

There is a lot of research work carried out to analyze the quality of online courses. The Babson Survey Research Group found that the number of students enrolling in online courses grew up to 5.8 million nationally in 2016 and one in four students took online courses in their higher education career. In higher education, the adoption of E-learning has expanded consistently over the past 13 years. As the number of online courses increases, the quality of the course is the main concern. In 2016, the federal government issued a set of suggestions to develop quality parameters for online learning. In this reviewing process, a learner's success rate of online course completion is also considered as an indicator. In most of the research work, the quality of the online course (well-designed online course) is analyzed with the help of these parameters such as effective communication, student active involvement in the content, timely feedback, course time period, motivation, and hands-on training (Schwab, 2017; Sharples \& Domingue, 2016; Tschorsch \& Scheuermann, 2016; Underwood, 2016).

Much research has been carried out on centralized review systems for various applications with different levels of trustworthiness and reliability. At present, there are many limitations in a current rating system as follows; due to the centralized system, the rating might be forged, with no audits from the third party and parity score. The centralized system is subjected to manipulating the reviews, which includes fake reviews for the promotion of the course, neglects the negative reviews, and the 
course ratings are not compared with other competitors in the E-learning platform. A centralized system can bias the choice of Subject Matter Experts (SME's) to influence the course's rating. These limitations can be addressed with the help of Blockchain Technology. Blockchain technology is an emerging technology; most systems are in the development stage and have not published any review systems for online courses.

Lina reviews (Lina 2018) is a platform for reviews that use blockchain technology to store- the reviews submitted by the users. It ensures the security and integrity of the reviews and rewarding the users for the review. In this platform, any company can build its system free of cost for participation. However, the system maintenance fee is charged by the platform. Lina platform uses a hybrid architecture (Public and Private) and a token is called Lina core which is used to store the detailed transactions. The main advantage of this platform is to reduce the high transaction fees imposed by the Ethereum Platform.

The first blockchain platform developed for the dental service review system is called Dentacoins (Dentacoin 2018). In this platform, the dentist can register their hospital in the system, which is reviewed by the public. The patients can provide reviews about the dentists based on their personal experience. Additionally, the trustworthiness of the system is ensured with the help of a trusted review system. The trusted review concept is introduced in this platform which is different from the traditional review system. In this system, the dentist mails the review link to the verified patients; only the verified patients can post the dental service review, whereas anyone can post the reviews in the traditional review system. All these steps are carried out using Ethereum blockchain through smart contracts using Dentacoin; both patients and dentists are rewarded with a token. The patients and dentist need to register, and the platform verifies the legitimacy of these participants. In the future, the Dentacoin platform aims to offer dental insurance and maintain the highly secure decentralized healthcare database of patients.

Zapit (2018) is a blockchain-based review platform mainly developed for Amazon's review system. It provides trust among Amazon sellers, consumers, and reviewers. Revain 2018 is IBM's Artificial intelligence-based review system. It mainly removes the fake reviews and the good quality reviews are stored on the Ethereum blockchain, where the reviews no longer tamper.

In the future, blockchain technology can be applied in innovative ways in the education industry. Blockchain technology greatly impacts designing the learning activities, formative evaluation, implementation, and keeping track of the whole online learning process. Kosba et al. (2016) discussed smart contracts' essentials on the Ethereum blockchain and its privacy preservation. Yuan and Wang (2018) presented a systematic analysis of blockchain and cryptocurrencies and discussed the technical advantages of bitcoin concerning the blockchain framework's six-layer reference model.

Androulaki et al. (2018) discussed the Hyperledger fabric architecture, the logic behind the design decision, and its implementation aspects of the distributed application programming model. Sukhwani et al. (2018) developed the stochastic model for Hyperledger Fabric and analyzed the full system's transaction details. The hierarchical model for Hyperledger fabric v1.4 was developed and 
its two timeout constraints are considered to measure the throughput of the platforms, estimated rejection probability of the transaction, and mean response delay of the transaction (Jiang et al. 2020).

A lot of research work is carried out on blockchain technology to extend its application to non-financial fields such as healthcare, education (Skiba 2017; Sharples and Domingue 2016), transportation (Astarita et al., 2020), Internet of Things (Xu et al., 2018), agriculture, supply chain (Azzi and Chamoun 2019), economy (Swan 2015) finance, etc., The healthcare industries have shown more impact on the blockchain assisted applications. Blockchain technology plays a vital role in the future development of healthcare industries (Amin, 2020). Chen et al. 2018a, b) introduced the benefits and challenges of blockchain applications for education. Swan (2015) divided the application development of blockchain into three stages such as Blockchain 1.0, 2.0, 3.0. Blockchain version 1.0 discussed the deployment of cryptocurrencies. Blockchain 2.0 discussed non-financial applications such as stocks, loans, property, and smart contracts. Blockchain 3.0 (Tschorsch \& Scheuermann, 2016; Underwood, 2016) is an extensive application such as health, culture, government, science, etc.

At present, the review data is stored in a centralized database and the course rating is not compared with any other competitors in the E-learning platform. The proposed system onboard the content from various online content providers and rate the content through an independent SME' which led to a new approach of ranking various courses in the same domain using a decentralized Blockchain based system. The Online content provider (OCP) validates the SME credentials based upon publications of SME in last twelve months in form of blogs, research papers, projects handles, and social media posts. There are several criteria for SMS selection:

1) Blogs and Ranking: Most of the experts publish Blogs and Articles in the subject area of their expertise. A systematic approach of ascertaining a Blog ranking can be done by various open software such as ahrefs.com etc. If the Blogger is consistently rating above average for a period of 1 year, he / she can be selected as an independent SME.

2) Social Media posts: Most of the professional social media networks such as LinkedIn provide a social media profile strength assessment based on followers, relevance of posts and credentials earned in a particular stream or technology. These reports can be requested on demand from LinkedIn and it can act as highly credible resource for identifying the independent SME.

3) Research Papers: Subject Matter experts publish papers and patents on new ideas and market needs. These research companies provide ratings for individuals based on their work and consistent publications. This data can be used as an authentic source of information of identifying independent SME.

Design and architecture of blockchain-based education content ranking system

This section elaborates on the proposed system design and architecture for an online education ranking system and its implementation details using Hyperledger 
fabric chain code and Federated SME-based ranking. In this proposed system, the parity Score is used to evaluate the courses on the same topic offered by multiple vendors using a decentralized blockchain mechanism. Parity score is independent of user rating, thereby allowing new content/course to be ranked at par with existing courses. The course rating system depends on the independent SME panel, federated score, and the help of specific parameters agreed by the content providers.

\subsection{Application scenario}

The application set-up of the proposed E-learning rating system is shown in Fig. 1. The actors of the model are as follows,

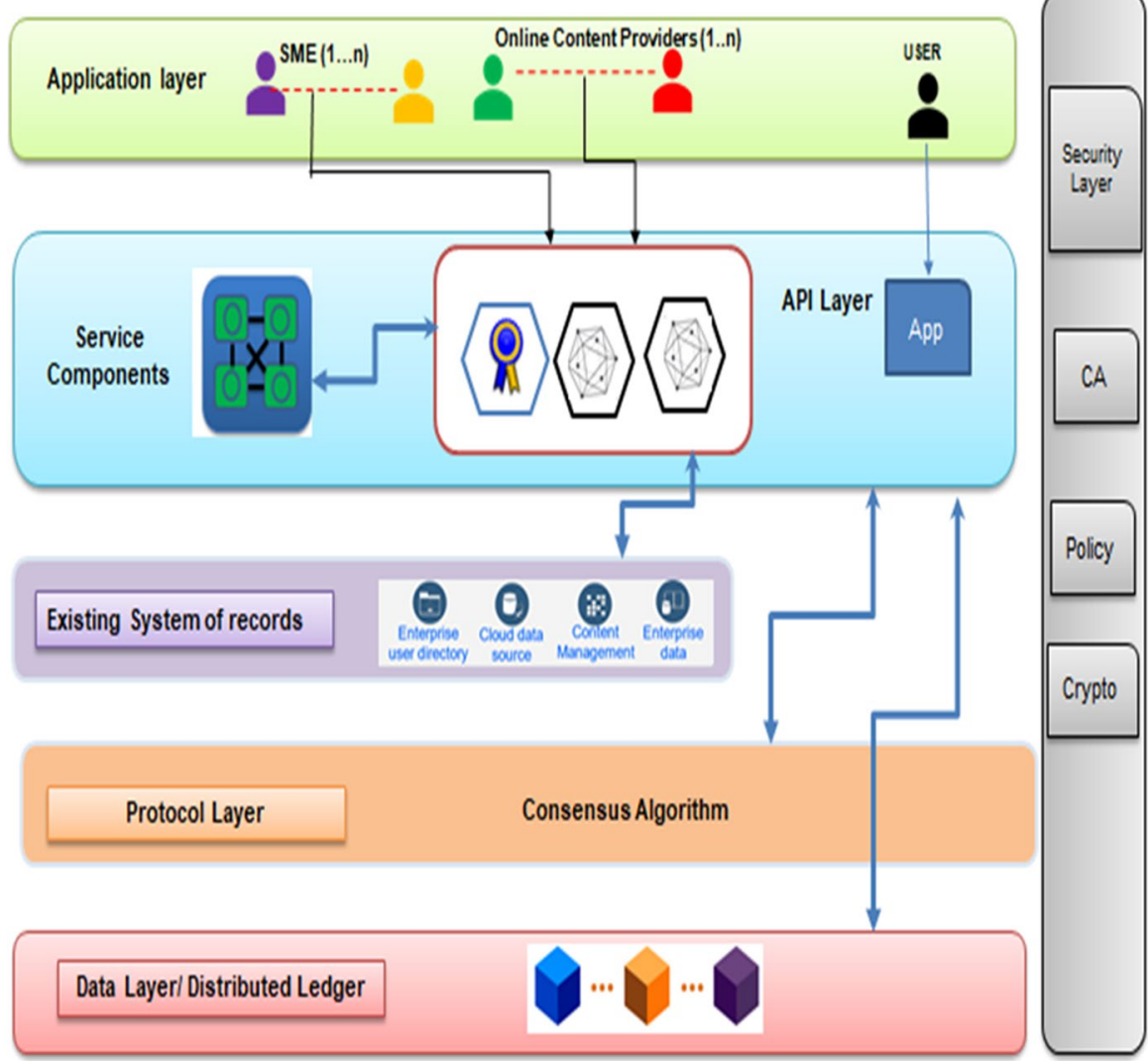

Fig. 1 Application Scenario of Proposed System 


\subsubsection{User}

The proposed online content rating system's unique feature is developed based on the Hyperledger fabric, a permissioned blockchain system. Each user can perform transactions with a blockchain network through API. A user who wants to pursue the online course needs to register with the Membership Service Provider (MSP)/ Fabric Certificate Authority. This feature allows only the registered user to participate in the system. The MSP validates the user and provides information about the user credentials and certificate for enrollment. The layer wise description of the Fig. 1 is as follows -

1) Application Layer-This layer hosts the mobile / web application that provides an interface for SME / Online content providers /users to initiate transactions on the Blockchain layer. These transactions are listed in detail in Sect. 4.2 of the document.

2) Service Components-These are some of the essential components of the Hyperledger Fabric Blockchain ecosystem. It includes Certification authority to issue valid credentials to users, Ordering services for distributing blocks and transactions and various API services to connect the layers below.

3) Existing System of records-This layer consists of an existing database of the online content providers that will be used by the Blockchain layer for providing the federated ratings.

4) Protocol Layer-This layer consists of various consensus algorithms used in Hyperledger fabric to ensure the consistency of data across various nodes and agreement of transactions by endorsing peers. This also includes system chaincodes used by endorsing peers, ordering services etc.

5) Data Layer-This layer consists of a distributed database used in the Blockchain system to keep the immutable trail of transactions so that any transaction can be verified and audited at any point of time. Generally this is a couch DB based world state ledger with the capabilities of a modern relational database.

\subsubsection{Subject matter experts}

Industrial experts (SME's) need to register on the same network as independent reviewers and validate the course content's quality. SME's review the content within the stipulated time period against specific parameters such as quality, duration of the course, ease of understanding, communication, practical orientation, industry relevance, and user level (Beginner, Intermediator, Advanced), etc. The registration of SMEs is vetted and endorsed by online content providers to ensure that all partners accept them in the consortium.

\subsubsection{Online content provider}

Online education provider companies such as Edureka, Coursera, Udmey, Edx, etc. can register on a Blockchain platform and push the information about all the courses (Al Harthy et al., 2019; Failed, 2019; Yuan \& Wang, 2018). The only registered user 
and SME can access the course content provided by the companies. Users can check the content, reviews, and ratings offered by legitimate learners who have completed the course. An online content provider can manage and update the entire course content based on the review comments.

\subsection{Design architecture and implementation details}

At present, the number of E-learning platforms is increasing day by day and incessantly creating many courses on the same topic. Many different online platforms offer similar courses; it is difficult for the course taker to choose the better one. In the present time, there is no mechanism for the user and independent SMEs to rank the courses independently based on their content, and the rating information is centrally managed with a monopoly. Many course takers have lost their interest in pursuing the course due to the fake reviews and poor course content. To ensure the security, integrity and prevent fake reviews and ranking of the online courses, there is a necessity of the immutable ledger, decentralized, transparent, and secure rating mechanism and the use of the most recent technologies to track the course at every level. From the points mentioned above, the secure online review system is very important to prevent the above limitations. Furthermore, the latest technology in computing that can handle privacy issues and provide an immutable ledger is Blockchain. Blockchain is a groundbreaking technology based on the consensus mechanism (Viriyasitavat \& Hoonsopon, 2019; Kraft, 2016)) introduced by Satoshi Nakamoto. It is mainly designed to maintain the transaction log for a renowned cryptocurrency named Bitcoin (Beck et al., 2016; Chung \& Kim, 2016; Collins, 2016).

In this paper, Hyperledger fabric is introduced for reviewing the online rating management system. The blockchain network's primary goal is to maintain the data in the distributed ledger in the form of a block where each block has multiple transactions. The transactions in the block are secured with the help of encryption and hash functions. The proposed online rating system is a service-orientated architecture for the online course content provider, which provides distributed ledger and chain code functionalities as a service. The design of the proposed system is as shown in Fig. 2. It shows the workflow of the proposed system:

In Steps 1 and 2, the user/SMEs need to get credentials to login into the fabric network. The login credentials are provided by a Membership Service Provider (MSP) in the fabric network. MSP is a component in a fabric network that defines rules to validate and authenticates the identities of users/SMEs and allows to access a network. MSP makes use of Fabric Certificate authority (CA) that verifies and revokes user certificates. In step 3, SMEs use a fabric SDK in order to communicate the review with the network. SMEs are issued with certificates from the CA in order to ensure the valid transaction is initiated over the network. When an SME communicates with the fabric network using Fabric client. In step 3, the fabric client makes a transaction proposal and signs the proposal with an SME certificate, and sends it to endorsing peers. In step 4, the endorsing peers verify the authorization of the proposal, if it passes, the peer generates the transaction 


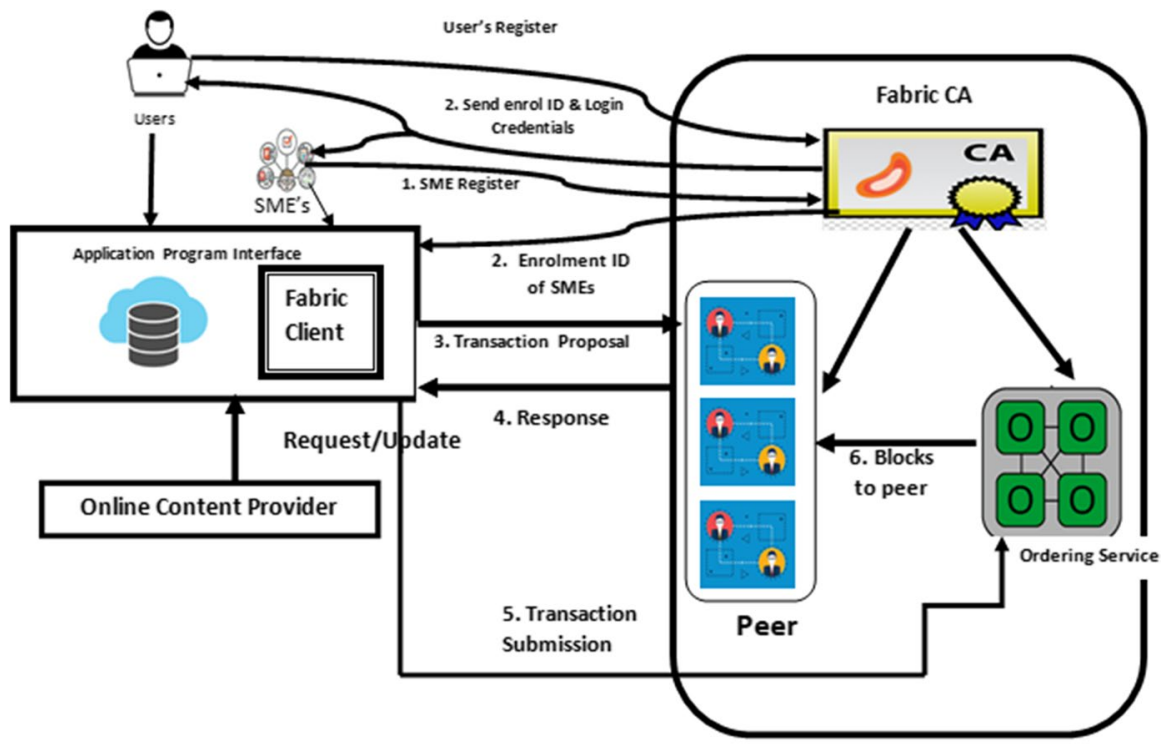

Hyperledger Fabric Network

Fig. 2 Design Architecture of the Proposed System

response using a certificate and shares the same with the Fabric client. The Fabric client collects and checks the response received from peers. In step 5, the fabric client shares the transaction proposal with the orderer. In step 6, Orderer orders the received transactions from the fabric client and generates a new block of ordered transactions, and signs the generated block with its certificate, and sends it to all peers.

The end-user (course taker/learner, independent SMEs, and online content provider) carried out the transaction through the front-end web application (Application program Interface), where the user and SME's can provide the reviews and rating of the course. Another unique feature of the proposed system is to ensure the integrity of the rating; independent SME's review the course content, prevent fake reviews, and provide the federated rating to the end-users. The federated rating is evaluated by averaging the ratings provided by independent impaneled SMEs and stored in a blockchain-based ledger to infuse trust amongst owners of different online learning platforms. Hyperledger fabric network has the following key components such as Fabric Certificate Authority (CA), Client application, peer, and ordering service. An application interacts with a blockchain network using the Fabric SDK. The Hyperledger Fabric SDK allows applications to interact with a Fabric blockchain network. It provides a simple API to submit transactions/Query to a ledger. The Fabric CA provides an identity for the users to perform transactions on the Hyperledger Fabric network. It also ensures the authenticity of the user and provides certificates to the users and SME's. The certificates are generated by a traditional certificate authority. The fabric client application has SDK to interact with Hyperledger fabric. The peer in the network maintains the ledger, smart contract, and events. The 
ordering service provides an ordered set of transactions and approves the inclusion of transactions into the blocks.

\subsubsection{Onboarding process}

There are two categories of onboarding required in the system. First, the onboarding of online education content providers, and second is the reviewer who is willing to provide ratings to the content.

There are two stages of onboarding for both the players. First, it obtains a valid digital certificate or a private key to participate in the network. The Blockchain components involved in this are Certification authority (CA) and Membership service provider (MSP). CA ensures that the valid certificate is given to the applicant, MSP ensures that the participant can enter with the valid credentials and suitable level of access based on his credentials. The service will use the existing LDAP, SAML, etc., mechanisms to authenticate and onboard the SMEs. The second component is the group of endorsers, validating if the content providers are pushing the correct category of content and the right set of reviewers are engaged in reviewing the respective content.

\subsubsection{Smart contract}

The smart contract is prominent in the blockchain network (Roszkowska, 2013). Smart contract or Chain code/business logic is a line of code (predefined set of rules) that enforces the agreement between the two parties without any third party involvement. When the specific condition is met, it automatically triggers the smart contract. The smart contracts are secured from the deletion and tampering because it is hoarded and managed in the blockchain platform (Lizcano et al., 2019). In Hyperledger Fabric-based solution, Smart contract is called Chain-code and is executed at the transaction endorsement stage. The typical cycle includes transactions to be initiated from the UI or Client application, and designated nodes called Endorsers run the smart contract to check the validity of the transactions, and if they are found to be valid, the transactions along with the endorsement policies are sent to ordering services for distribution across the network. There are system chain-codes at orderers and committers that ensure that transactions are ordered correctly and ledgers at all nodes are updated with the same state of data (Lizcano et al., 2019).

With context to the proposed method, the smart contract (chain code) process is categorized into two-step which is detailed as follows,

SME On-board Smart Contract The online boarding process of SME is shown in Fig. 3. The on-boarding of SMEs process is mentioned in the sequence 1,2,3,4. In step 1, SME's register themselves in a blockchain network. SME's of the specific areas can nominate themselves to become part of the Blockchain network and present their primary credentials and social media credentials. In steps 2 and 3, the endorser node receives this transaction of SME onboarding, it allows various organizations consisting of online content providers to review and vote for an SME to be onboarded. In step 4, based on the majority vote decision, the SME is accepted or 


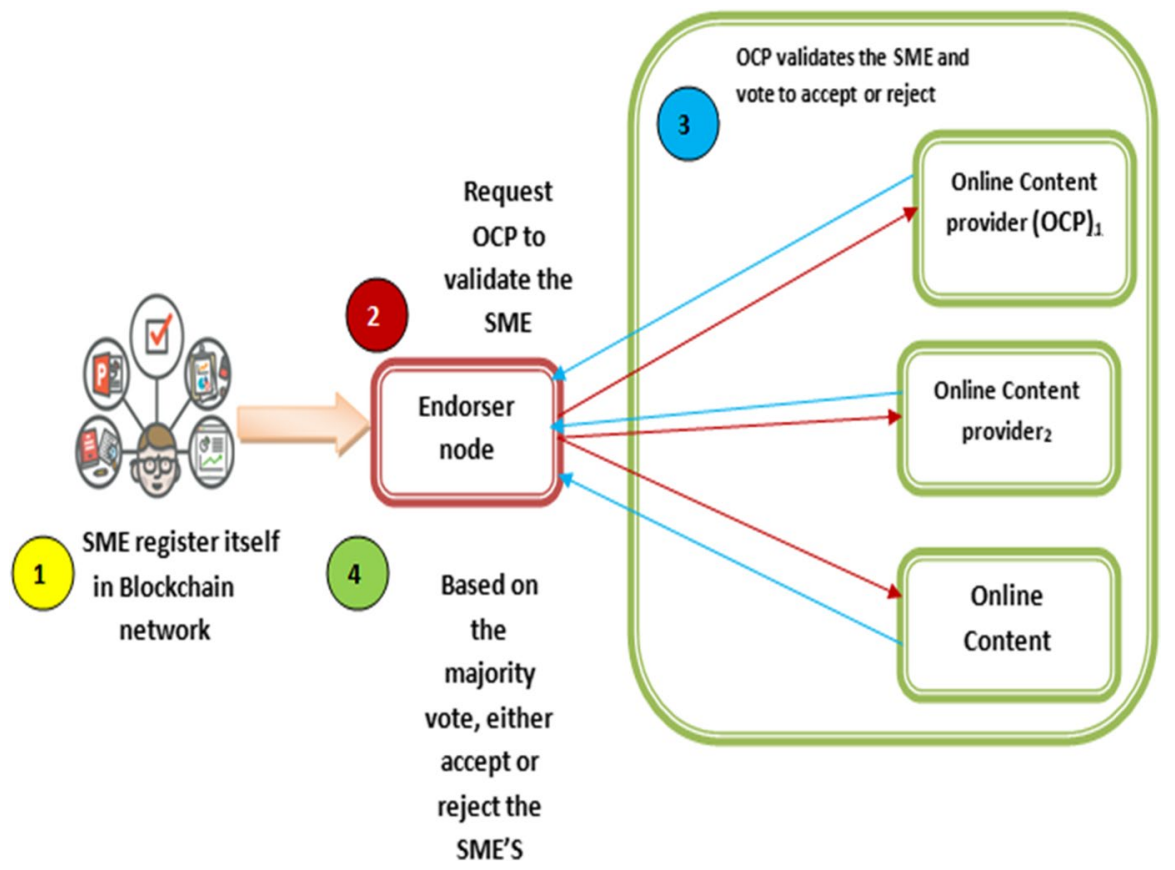

Fig. 3 SME onboarding process

rejected in the system. This ensures that there is no bias towards a particular person or entity, and hence the decision-making will be unbiased.

Rating Submissions by SME Once the new content is pushed for a particular area (e.g., Java, Machine Learning, etc.), SME's get the notification to review the content and provide the rating. A smart contract is executed to allow the federated rating collection and aggregation mechanism presented in Sect. 4.2.2. The smart contract takes care of valid SMEs reviewing the respective content and ratings based on the prescribed format only with correct aggregation being done.

\subsubsection{Transaction flow}

The Hyperledger fabric framework is primarily used for providing a distributed Blockchain-based rating system in which all the transactions are secure, transparent, immutable, and audit compliant. The proposed Hyperledger fabric network is deployed in a Docker container. The fabric network provides for Certificate Authority (CA), Orderer, and peers and datastores, namely World state and Blockchain.

The client application will have an SME interface, a Content Provider interface, and an admin interface. The interface provides a mechanism to log in to the application and input the desired functionality. The SME interface provides a connection with an application to record the feedback about the course by the SME, stored securely in a blockchain-based ledger. Similarly, the content provider interface 
allows the online learning content providers to upload their contents and secure access for SME review.

The CA provides authentication and validation of user identities and their designated roles and access permissions on the blockchain network. The orderer helps to sequence the incoming transactions from different SMEs and processes logically lined up for execution. The system utilizes a NoOps based solo consensus mechanism.

After being authenticated by the consortium members, the SMEs are onboarded and given access to the course to be rated. The individual ratings of SME are stored in a Blockchain-based ledger to ensure that they are not tampered with; once the consortium approved SMEs have recorded their individual ratings, the Federated rating mechanism kicks into generating federated ratings taking into account all SME ratings.

The transaction flow of the Hyperledger framework is as shown in Fig. 4. In step 0, SME's receives notification from the Online Content provider to review the relevant content. The SME reviews the content based on the parameters like Quality of content, Understandability, Existing User Rating (if any), Duration of course, Availability of Hands-on labs, Industry relevance of the course content (Beginner, Intermediate and Expert) and Availability of Evaluation methods in course. In step 1.1 \& 2.1, the SME submits the ranking to the Endorser Peer in the form of a transaction. In step 1.3 and 2.3, the Endorser has an encoded business process, termed as Smart Contract (Chain code) for validating the ranking and calculating the Federated rating (FR) based on the submitted ranking. The Smart contract for calculating the FR uses weighted formula approach for calculating the FR. The idea of using federated rating helps in aggregating the rankings received from different SMEs for the same course, hence normalizing and bringing parity to the ranking of any course. The weighted formula offers more weightage to parameters namely Availability of evaluation methods, Industry relevance of the courses, Availability of hands on labs

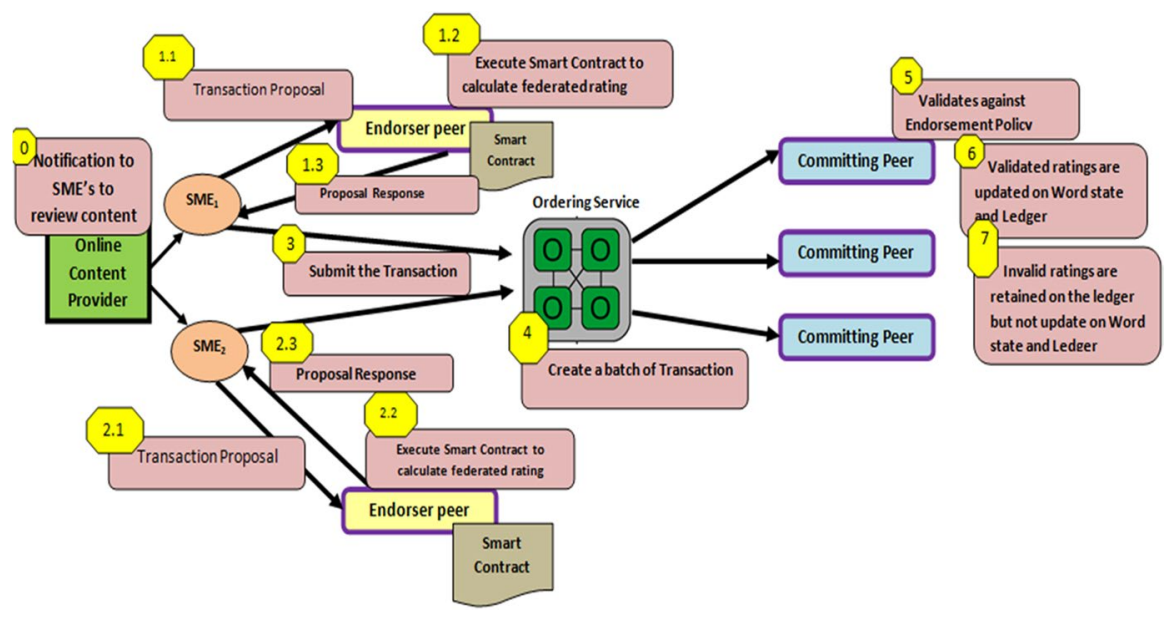

Fig. 4 Transaction flow of the proposed system 
compared to the remaining ones. In step 3, the proposed response of Endorser is shared with the client application. The client receives sufficient endorsements and it verifies the endorsement policies and submits the transaction to the ordering service in step 4. In step 5, the ordering service ordered the set of transactions and delivered to the committing peers in the network. In step 6 , the committing peer validates the transaction against the endorsement policies and the validated ratings are updated on world state and ledger in step 7.

\subsubsection{Federated rating mechanism and algorithm using blockchain}

The paper proposes a rating mechanism to enhance unbiased, trustworthy, and fair ratings to the courses available across different courses offered by different online learning platforms. The rating offered by independent impaneled SMEs will be federated by agreeing to an established model and code of ethics. The federated rating (FR) will be drawn by using a weighted approach based on importance assigned to parameters making the rating relevant (Dimpal et al et al.,). The ranking by the SME is based on the parameters like Quality of content, Understandability, Existing User Rating(if any), Duration of course, Availability of Hands-on labs, Industry relevance of the course content(Beginner, Intermediate and Expert), and Availability of Evaluation methods in the course. The idea of using federated rating helps in aggregating the rankings received from different SMEs for the same course, hence normalizing and bringing parity to the ranking of any course. The Federated Ratings are based on the weighted aggregation of the ranking provided by independent empanelled SMEs and are stored in a blockchain-based ledger to infuse trust amongst owners of different online learning platforms. The Blockchain-based ledger will provide trust against any manipulations of rating, fudging of data, and bring transparency to the overall rating system. The federated rating approach will also help newly created content with independent ratings irrespective of the number of subscribers, which is the current benchmark for course ratings. Fig. 5. Shows the federated rating algorithm. The input for the Federated rating algorithms is SME's address or Identity, and rating of the course provided by SME's. The endorser executes the smart contract and computes the average federated rating values of SME's. The proposed response of Endorser is shared with the client application. The client receives sufficient endorsements and it verifies the endorsement policies and submits the transaction to the ordering service as mentioned in Fig. 4.

\section{Result and security analysis}

In this section, the proposed online reviewing system's work communicating with the Blockchain platform is discussed. The prototype of the proposed system is developed on the Hyperledger Fabric platform. The client user interface is given to all the system participants to initiate the transaction once their identity is validated. The participants can communicate and store the transaction in the blockchain network through the REST server composer. One of the major features of the network is to design the smart contract for the rating of the course easily and efficiently. The 
Fig. 5 Federated rating Algorithm

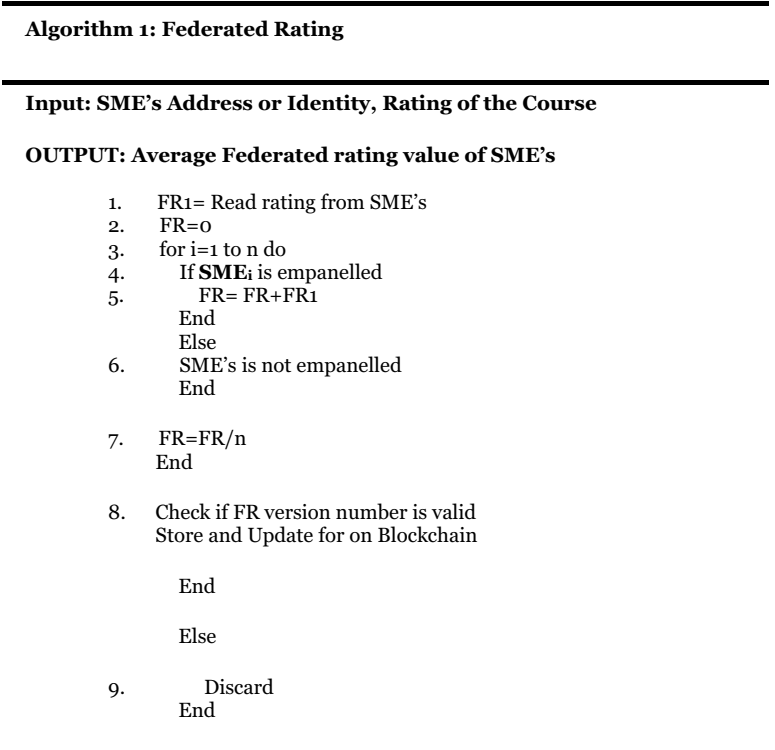

smart contract in a Hyperledger based network consists of four major components; defining the participants, script for reviewing the course, access control rule security and permission, and queries and accessing the database. Figure 6. shows the participant's definition in the hyperledger fabric. The participants of the proposed systems are SME, content Provider, and course name. The assets of the proposed system are ratings offered by SMEs and federated rating.

The main important things to define while designing a blockchain network using Hyperledger fabrics are the participant's definition, smart contract functions, access control policies, etc. Hyperleder Fabric use chaincode (smart contract) functions or scripts to register and update any transaction that happens in the system and upon successful execution stores it in the immutable Blockchain ledger. The script function in Fig 7 is an example of one such function where the content ranking of the course is updated post the review from SME (Caliper 2019). The content evaluation criteria is already mentioned in Fig. 5 and script functions aim to codify the

Fig. 6 Participants definition in the Hyperledger fabric

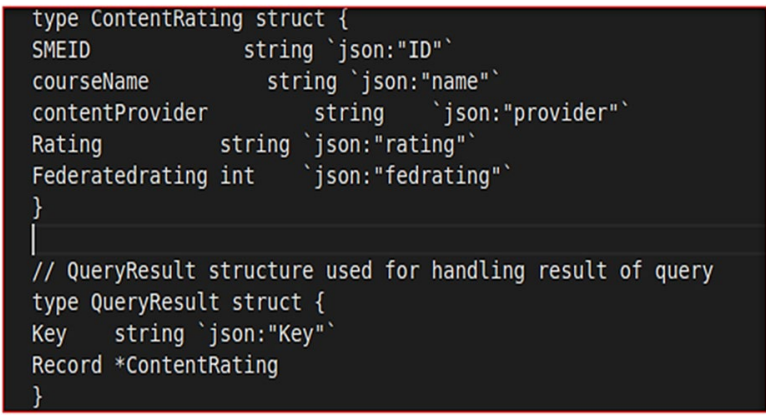


Fig. 7 Script file function to update transaction

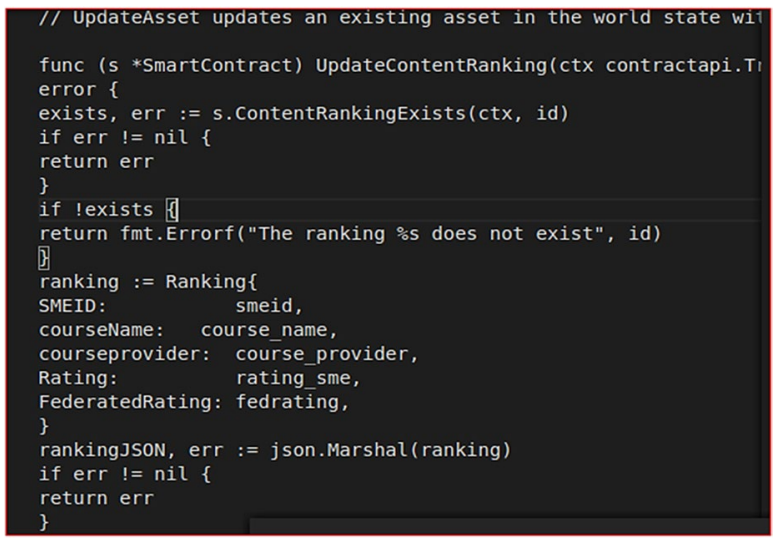

business logic into the code that can update and commit the transaction in the system. Some other script functions could be - Register SME, Register New Course, Evaluate Course, Revise Federated Ranking etc. This transaction function is mainly for updating the rating in the world state. This script file contains the function related to updating, and deleting the rating in the blockchain-based network. The client application is shown in Fig. 8 for an online review system where Online Content Providers can update and delete the course content and SME can provide the review in the blockchain network.

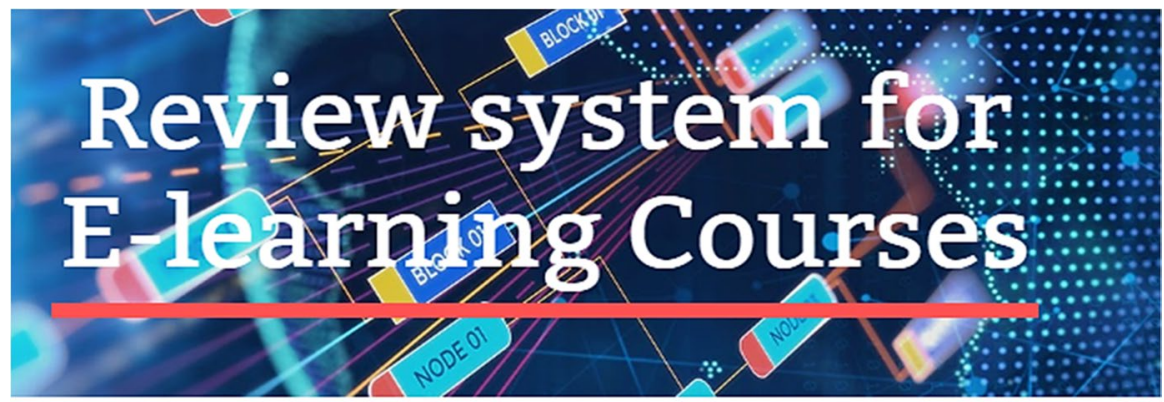

Client

Content Updating

Fig. 8 Client Application for Online review system 
The online content providers can give notification to the suitable SME' to review the course content and store the federated ranking in the Blockchain The SME's can use this web portal to view and review the course content to be shown in Fig. 9. The SME's review management portal allows the SME's to view the course content, update the SME's profile, and rate the course content with the user interface's help. The online content providers update the course information on the existing course content based on the SME's reviews and send the review request through the user interface to the Blockchain network.

The proposed system's novelty is to provide decentralized online review systems to validate the rating's trustworthiness and enables the consortium-based onboarding of Subject Matter Experts (SME's) endorsed by the stakeholders. This system ensures a secure and transparent review system where one can provide fake reviews, it prevents fake reviews. A smart contract designed to calculate the course content's federated rating provided SME's and updates the results in the Blockchain network. Once the federated rating is finalized, the results are notified to the SME's and online content providers.

The very fundamental and nature of a Blockchain network is good enough to establish the fact that the submitted reviews cannot be manipulated. Some of reasons are-

1) All reviews and transactions are hashed, signed, and added to the chain that forms immutable group of Blocks protected by hashes.

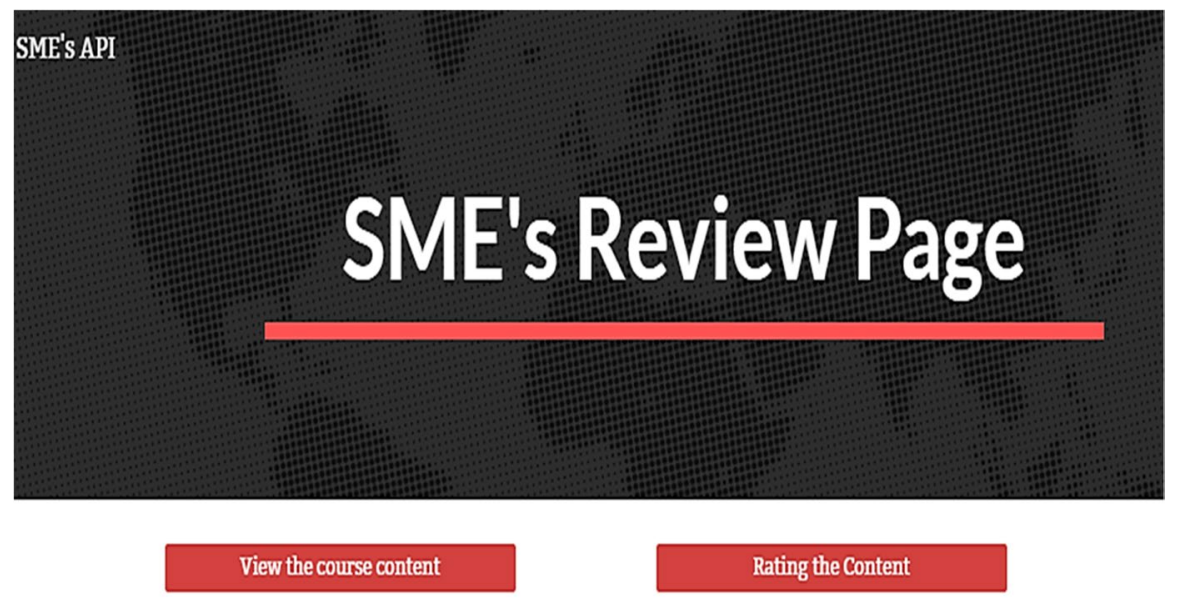

Update the SUE's profile

Fig. 9 SME's web portal 
2) Because of the decentralized nature of data multiple nodes will hold the copy of ledger and any attempt to modify / corrupt information will lead to instant correction unless $>50 \%$ nodes are compromised which is highly unlikely.

3) The solution uses Hyperledger Fabric as a solution, and it has endorsement process that is coupled with chain-code (smart contract). This ensures that review process transaction is verified by all concerned parties before this becomes part of the system.

4) Above three points are good enough to proof that any kind of review rating manipulation is impossible with Blockchain based system.

\subsection{Security against manipulation of SME's Assignment}

At present, the ratings are stored in the centralized system in which the selection or nomination of SMEs can be biased. It will affect the fairness of the ratings by assigning his/her own choice of SME's to get preferred results. The proposed system ensures the security, integrity and prevents fake reviews and ranking of the online courses.

Proof Sketch The proposed system provides a consortium-based onboarding of SME's endorsed by all the online content providers. Once the onboarding transaction request submitted by the SME's goes through consensus, various online content providers validate and vote for SME's. The endorsement policies ensure that there is no bias towards an SME's onboarding process.

Security against manipulation of submitted reviews

Any ranking agency that is centralized or monopolized will not trust all the content vendors since the rankings can be manipulated and data can be fudged. Hence the Blockchain implementation with Federated SME ranking process can resolve this issue.

Proof Sketch In this proposed system, the rating computed by the SME's of the course is stored in Blockchain through a decentralized consensus algorithm and cryptography. It is impossible to manipulate the data due to a large amount of computing power that will be needed. Blockchain technology ensures the security and privacy of the data. In the present system, the ratings are stored in centralized servers that are the target of attackers.

\section{Conclusion}

Blockchain platforms are the cornerstone for designing decentralized systems that can serve a different range of applications. It changed the traditional way of storing the data to a new decentralized, robust, secure, and transparent. The novelty of the proposed system is to provide decentralized online review systems to validate the rating's trustworthiness and enables the consortium based onboarding of Subject Matter Experts (SME's) endorsed by the stakeholders. This system ensures a secure 
and transparent review system where no one can provide fake reviews. Our proposed system provides a clear view to the learners to identify the course's quality offered by different online content providers using the federated rating. The course's federated rating helps to decrease the disparity in the course contents made available by different vendors on the same topic. Simultaneously, online education companies get a clear view of the improvement needed to uplift the ranks and make it popular. In future work, the machine learning-based recommendation engine will be integrated with the proposed system to decide the course's category based on the level of learners (Basic/Intermediate/Advanced), and the parity score for the course will be generated based on the weighted formula.

\section{Declarations}

Conflicts of interests The authors have no conflict of interest.

Competing interests No competing interest for the work.

\section{References}

Al Harthy, K., Al Shuhaimi, F., \& Al Ismaily, K. K. J. (2019). The upcoming blockchain adoption in higher-education: Requirements and process. In 2019 4th MEC international conference on big data and smart city (ICBDSC) (pp. 1-5). IEEE. https://www.semanticscholar.org/paper/The-upcomingBlockchain-adoption-in-requirements-Harthy-Shuhaimi/804e8aee81e2e23ed0ededfb9f198e4095e 81496

Ali Alammary, Samah Alhazmi, Marwah Almasri and Saira Gillani (2019). Blockchain-Based Applications in Education:A Systematic Review. Applied Science 9(2004). https://www.mdpi.com/20763417/9/12/2400?_cf_chl_captcha_tk_=luxNFaoVcups.K6wglgzdsnY2QBe_049dfYvvSxF7kw1636454371-0-gaNycGzNCdE

Amin, A. D. (2020). Blockchain technology in banking and finance sector: Its effects and challenges. CARE J., 31(349-358), 24.

Androulaki, E., Barger, A., Bortnikov, V., Cachin, C., Christidis, K., De Caro, A., Enyeart, D., Ferris, C., Laventman, G., Manevich, Y., Muralidharan, S., Murthy, C., Nguyen, B., Sethi, M., Singh, G., Smith, K., Sorniotti, A., Stathakopoulou, C., Vukolic, M., ... Yellick, J. (2018). Hyperledger fabric: a distributed operating system for permissioned blockchains. EuroSys, 30(1-30), 15.

Astarita, V., Giofrè, V. P., \& Mirabelli, G. (2020). Solina, V (2020) A Review of Blockchain-Based Systems in Transportation. Information, 11, 21.

Azzi, R., \& Chamoun, R. K. (2019). Sokhn, M (2019) The power of a blockchain-based supply chain. Computers \& Industrial Engineering, 135, 582-592.

Beck, R., Czepluch, J. S., Lollike, N., Malone, S. (2016). Blockchain - The Gateway to Trust-Free Cryptographic Transactions. In Research Papers from ECIS2016, (Istanbul, 2016). https://pure.itu.dk/ portal/en/publications/blockchain--the-gateway-to-trustfree-cryptographic-transactions(c0965a66038d-456f-b1c2-bb32282bd28a)/export.html

Cachin, C. (2016). Architecture of the hyperledger blockchain fabric. In Proceedings of the Workshop on Distributed Cryptocurrencies and Consensus Ledgers, Hangzhou, China, 11-13 May 2016; vol. 310, p. 4. https://www.scirp.org/(S(czeh2tfqw2orz553k1w0r45))/reference/referencespapers.aspx? referenceid $=2723156$

Caliper (2019) H. Hyperledger Caliper Architecture. Available online: https://hyperledger.github.io/calip er/docs/2_Architecture.html (accessed on 10 March 2019) 
Fran Casino, Thomas K. Dasaklis, Constantinos Patsakis.A systematic literature review of blockchainbased applications: Current status, classification and open issues, Telematics and Informatics, 36,2019.pp. 55-81, https://doi.org/10.1016/j.tele.2018.11.006

Chen, G., Xu, B., Lu, M., et al. (2018a). Exploring blockchain technology and its potential applications for education. Smart Learn. Environ., 5, 1. https://doi.org/10.1186/s40561-017-0050-x

Chen, G., Xu, B., Lu, M., \& Chen, N. (2018b). S (2018) Exploring blockchain technology and its potential applications for education. Smart Learn. Environ., 5, 1.

Chung, M., \& Kim, J. (2016). The internet information and technology research directions based on the fourth industrial revolution. KSII Transactions on Internet and Information Systems, 10(3), 1311-1320.

Collins, R. (2016). Blockchain: A new architecture for digital content. Econtent, 39(8), 22-23.

Dentacoin 2018. Dentacoin: The Blockchain Solution for the Global Dental Industry. https://denta coin.com/web/whitepaper/Whitepaper-en1.pdf. Accessed 22 Apr 2018

Devine, P. (2015). Blockchain learning: can crypto-currency methods be appropriated to enhance online learning? Presented at the ALT Online Winter Conference 2015, Online (United Kingdom). http://oro.open.ac.uk/44966/

C. Li, J. Guo, G. Zhang, Y. Wang, Y. Sun and R. Bie, "A Blockchain System for E-Learning Assessment and Certification," 2019 IEEE International Conference on Smart Internet of Things (SmartIoT), Tianjin, China, 2019, pp. 212-219, https://doi.org/10.1109/SmartIoT.2019.00040.

Fanning, K., \& Centers, D. P. (2016). Blockchain and its coming impact on financial services. J. Corp. Account. Finance, 27(5), 53-57. https://doi.org/10.1002/jcaf.22179

Fernández-Caramés, T. M., \& Fraga-Lamas, P. (2019). Towards next-generation teaching, learning, and context-aware applications for higher education: A review on blockchain, IoT, fog and edge computing enabled smart campuses and universities. Applied Sciences, 9(21), 4479.

Haber, S., \& Stornetta, W. S. (1991). How to time-stamp a digital document. Journal of Cryptology, 3(2), 99-111. https://doi.org/10.1007/BF00196791

MB Hoy, An introduction to the Blockchain and its implications for libraries and medicine. Med. Ref. Serv. Q. 36(3), 273-279 (2017) https://doi.org/10.1080/02763869.2017.1332261

https://www.marketsandmarkets.com/Market-Reports/blockchain-technology-market-90100890.html

Hyperledger. Hyperledger Blockchain. Available online: https://www.hyperledger.org/ (accessed on 10 January 2020)

Hyperledger. Hyperledger Caliper. Available online: https://www.hyperledger.org/projects/caliper/ (accessed on 5 January 2019).

Jain, Dimpal, et al. (2016). Selection and ranking of E-learning websites using weighted distancebased approximation. Journal of Computers in Education, 3, 193-207.

Jansen, Darco; Rosewell, Jonathan and Kear, Karen (2017). Quality Frameworks for MOOCs. In: Jemni, Mohamed; Kinshuk and Khribi, Mohamed Koutheair eds. Open Education: from OERs to MOOCs. Lecture Notes in Educational Technology. Berlin Heidelberg: Springer, pp. 261281. https://link.springer.com/chapter/10.1007/978-3-662-52925-6_14

Jiang, L., Chang, X., Liu, Y., et al. (2020). Performance analysis of Hyperledger Fabric platform: A hierarchical model approach. Peer-to-Peer Netw. Appl., 13, 1014-1025. https://doi.org/10.1007/ s12083-019-00850-z

Kosba AE, Miller A, Shi E, Wen Z (2016) charalampos papamanthou: Hawk: The blockchain model of cryptography and privacy-preserving smart contracts. IEEE Symposium on Security and Privacy:839-858. https://ieeexplore.ieee.org/document/7546538

A Kosiba, A Miller, E Shi, Z Wen, C Papamanthou, in 2016 IEEE Symposium on Security and Privacy (SP). Hawk: The Blockchain Model of Cryptography and Privacy-Preserving Smart Contracts (2016), pp. 839-858 https://doi.org/10.1109/SP.2016.55

Kraft, D. (2016). Difficulty control for blockchain-based consensus systems. Peer Peer Netw. Appl., 9(2), 397-413. https://doi.org/10.1007/s12083-015-0347-x

Lina.Review: Blockchain Based Review Platform, LinaNetwork, January 1st, 2018. [Online]. Available: https://lina.review/lina_whitepaper.pdf [Accessed: 22- Apr- 2018]

Lizcano, D., Lara, J. A., White, B., \& Aljawarneh, S. (2019). Blockchain-based approach to create a model of trust in open and ubiquitous higher education. Journal of Computing in Higher Education, 32, 1-26.

MathSciNet Article Google Scholar

Nakamoto, S. Bitcoin: A Peer-to-Peer Electronic Cash System. 2008. Available online: https://bitcoin. org/bitcoin.pdf (accessed on 12 September 2019) 
Peck, M. (2016). A blockchain currency that beat s bitcoin on privacy. IEEE Spectrum, 53(12), 11-13. https://doi.org/10.1109/MSPEC.2016.7761864

Revain 2018. New generation feedback platform based on the blockchain technology. Revain Whitepaper. [Online]. Available: http://revain.org/pdf/wp/en-wp.pdf

Roszkowska, E. (2013). "Rank Ordering Criteria Weighting Methods - a Comparative Overview." https://www.researchgate.net/publication/280081585_Rank_Ordering_Criteria_Weigh ting_Methods_-_A_Comparative_Overview

Schwab, K. (2017). The Fourth Industrial Revolution. The Crown Publishing Group.

Shaker, M., Shams Aliee, F., \& Fotohi, R. (2021). Online rating system development using blockchain-based distributed ledger technology. Wireless Networks. https://doi.org/10.1007/ s11276-020-02514-w

Sharples, M., Domingue, J,. (2016). The blockchain and kudos: A dstributed system for educational record, reputation and reward. Adaptive and adaptable learning (Springer, Cham), pp. 490-496. https://doi.org/10.1007/978-3-319-45153-4_48

Skiba, D. J. (2017). The potential of Blockchain in education and health care. Nursing Education Perspectives, 38(4), 220-221. https://doi.org/10.1097/01.NEP.0000000000000190

Sukhwani, H., Wang, N., Trivedi, K. S., \& Rindos, A. (2018). Performance modeling of Hyperledger Fabric (permissioned blockchain network). NCA: 1-8. https://www.researchgate.net/publication/ 329298342_Performance_Modeling_of_Hyperledger_Fabric_Permissioned_Blockchain_Network

Sun, X., Zou, J., Li, L., et al. (2021). A blockchain-based online language learning system. Telecommunication Systems, 76, 155-166. https://doi.org/10.1007/s11235-020-00699

SUN, Han; WANG, Xiaoyue; WANG, Xinge. Application of Blockchain Technology in Online Education. International Journal of Emerging Technologies in Learning (iJET).13(10), pp. 252-259, oct. 2018. ISSN 1863-0383. Available at: https://online-journals.org/index.php/i-jet/article/view/9455. Date accessed: 12 Mar. 2021. https://doi.org/10.3991/ijet.v13i10.9455.

M Swan (2015) Blockchain: Blueprint for a New Economy, 1st edn. (O’Reilly Media, Sebastopol, CA, 2015)

Tschorsch, F., \& Scheuermann, B. (2016). Bitcoin and beyond: A technical survey on decentralized digital currencies. IEEE Commun. Surv. Tutorials, 18(3), 2084-2123. https://doi.org/10.1109/COMST. 2016.2535718

Underwood, S. (2016). Blockchain beyond Bitcoin. Communications of the ACM, 59(11), 15-17. https:// doi.org/10.1145/2994581

Viriyasitavat, W., \& Hoonsopon, D. (2019). Blockchain characteristics and consensus in modern business processes. Journal of Industrial Information Integration, 13, 32-39.

M Vukolić (2015) The Quest for Scalable Blockchain Fabric: Proof-of-Work vs. BFT Replication. Open problems in network security (Springer, Cham, 2015), pp. 112-125 https://doi.org/10.1007/978-3319-39028-4_917.

Williams, P. (2019). Does competency-based education with blockchain signal a new mission for universities? Journal of Higher Education Policy and Management, 41(1), 104-117.

Xu, Q., Aung, K. M. M., Zhu, Y., \& Yong, K. L. (2018). A blockchain-based storage system for data analytics in the Internet of things. In New Advances in the Internet of Things; Springer: Berlin. Germany, 2018, 119-138.

Yuan, Y., \& Wang, F.-Y. (2018). Blockchain and cryptocurrencies: Model, techniques, and applications. IEEE Trans Systems, Man, and Cybernetics: Systems, 48(9), 1421-1428.

Zapit 2018. Understanding Zapit: The only blockchain-verified product reviews platform. https://zapit. online/Zapit_Whitepaper-v0.6.pdf. Accessed 22 Apr 2018

Publisher's note Springer Nature remains neutral with regard to jurisdictional claims in published maps and institutional affiliations. 


\section{Authors and Affiliations}

\section{Anuj Garg ${ }^{1}$ - Sharmila A² Pramod Kumar $^{3}$ - Mani Madhukar ${ }^{4}$. Octavio Loyola-González ${ }^{5} \cdot$ Manoj Kumar $^{6}$ (D)}

Anuj Garg

garg.anuj@in.ibm.com

Sharmila A

sharmila1ece@gmail.com

Pramod Kumar

tulasacademic@gmail.com

Mani Madhukar

manimad9@in.ibm.com

Octavio Loyola-González

olg@altair.consulting

1 Innovation and Transformation Leader, IBM India Pvt. Ltd, Bangalore, India

2 Electronics and Communication Engineering, Krishna Engineering College, Mohan Nagar, Ghaziabad 201007, Uttar Pradesh, India

3 Computer Science Engineering, Krishna Engineering College, Mohan Nagar, Ghaziabad 201007, Uttar Pradesh, India

4 Program Manager, IBM India Pvt Ltd., Bangalore, India

5 Altair Management Consultants, Calle de José Ortega y Gasset 22-24, 5th floor, 28006 Madrid, Spain

6 School of Computer Science, University of Petroleum and Energy Studies, Dehradun, India 